\title{
A relationship between trees and Kelly-Mac Lane graphs
}

\author{
Eugenia Cheng \\ Department of Pure Mathematics, University of Cambridge \\ E-mail: e.cheng@dpmms.cam.ac.uk
}

October 2002

\begin{abstract}
We give a precise description of combed trees in terms of KellyMac Lane graphs. We show that any combed tree is uniquely expressed as an allowable Kelly-Mac Lane graph of a certain shape. Conversely, we show that any such Kelly-Mac Lane graph uniquely defines a combed tree.
\end{abstract}

\section{Contents}

Introduction 2

1 Backoround on Kelly-Mac Lane Graphs 3 1.1 Shapes . . . . . . . . . . . . . . . . . . . . 3

1.2 Graphs. . . . . . . . . . . . . . . . . . . . 4

1.3 Allowable morphisms . . . . . . . . . . . . . . . . 6

1.4 Duality . . . . . . . . . . . . . . . . . 7

\begin{tabular}{|lll}
2 & Informal description of trees & 7
\end{tabular}

3 Formal description of trees $\quad 8$

4 Trees as morphisms in $K 1 \quad 12$

5 Composition of trees $\quad 15$

6 The graph of a tree is allowable 20

7 Everv allowable graph is a tree 23

$\begin{array}{ll}\text { References } & 26\end{array}$ 


\section{Introduction}

In this paper we show how trees may be expressed as allowable KellyMac Lane graphs of a certain shape.

The trees in question are those arising in [1] and 4] to express configurations for composing higher-dimensional cells in the theory of opetopic $n$-categories. Kelly-Mac Lane graphs are introduced in [8] to study coherence for symmetric monoidal closed categories. The main result of this paper states that a tree is precisely an allowable Kelly-Mac Lane graph of shape

$$
X_{m_{1}} \otimes \cdots \otimes X_{m_{k}} \longrightarrow X_{\left(\sum_{i} m_{i}-k+1\right)}
$$

where each $m_{i} \geq 0$ and for any $m \geq 0$ we write

$$
X_{m}=\left[1^{\otimes m}, 1\right] .
$$

We begin, in Section 1 by giving a minimal account of the theory of Kelly-Mac Lane graphs, including no more than what is required for the purposes of this paper. We refer the reader to $[\underline{8}$ for the full details, noting that for the purposes of this work we consider only the strict monoidal case.

We then recall the trees in question, as defined in [4. We give an informal description in Section 2, and in Section 3 the formal description that paves the way for the ensuing results. In Section 4 we show how to express a tree as a graph, not a priori allowable, and in Section [5] we characterise tree composition in this new framework.

This enables us to prove, in Section 6, that the graph of a tree is allowable. Finally, in Section 7 we show that every allowable graph of the correct shape is a tree, giving the main result of this work.

\section{Further work}

1) The trees in question arise in the 'slice' construction described in [1] and 4] in the construction of opetopes and definition of opetopic $n$ category. Thus the new way of expressing trees as described in this paper can be used to give a description of the category of opetopes; this is described in 3].

2) Blute(2] has established a relationship between Kelly-Mac Lane graphs and the proof nets of Linear Logic, so the material in the present work should in turn give a relationship between opetopes and proof nets.

3) Finally we note that the allowable Kelly-Mac Lane graphs are the morphisms of the free symmetric monoidal closed category on one object. Although we do not need to use this fact in this paper, it should give a more abstract approach to this material, the significance of which is currently unclear. 


\section{Acknowledgements}

This work was supported by a PhD grant from EPSRC. I would like to thank Martin Hyland and Tom Leinster for their support and guidance.

\section{Background on Kelly-Mac Lane Graphs}

In this section we give a brief account of the theory of Kelly-Mac Lane graphs. Note that we will only be concerned with the strict monoidal version for the purposes of this paper.

In [8], Kelly and Mac Lane study coherence for symmetric monoidal closed categories. In brief, a symmetric monoidal closed category is a symmetric monoidal category $\mathcal{C}=(\mathcal{C}, \otimes, I, a, b, c)$ equipped, in addition, with a functor

$$
[,]: \mathcal{C}^{\mathrm{op}} \times \mathcal{C} \longrightarrow \mathcal{C}
$$

and natural transformations

$$
\begin{aligned}
& d=d_{A B}: A \longrightarrow[B, A \otimes B] \\
& e=e_{A B}:[A, B] \otimes A \longrightarrow B
\end{aligned}
$$

satisfying certain axioms. (Here $a, b$ and $c$ are the natural isomorphisms for associativity, unit and symmetric action respectively.) In particular we have a natural isomorphism

$$
\pi: \mathcal{C}(A \otimes B, C) \longrightarrow \mathcal{C}(A,[B, C]) .
$$

Kelly and Mac Lane refer to such categories simply as closed categories and we do the same.

Kelly and Mac Lane introduce a notion of graph which enables a partial solution to the question: when does a diagram in a closed category commute? In fact we are not concerned with the coherence question here, so we only give the construction of the graphs and state one theorem from [8] which will later be useful.

Kelly and Mac Lane define a category $G$ whose objects are shapes and whose morphisms are graphs; this is seen to be a closed category. They then define a subcategory whose morphisms are the allowable morphisms. These are defined as precisely those morphisms of $G$ demanded by the symmetric monoidal closed structure.

\subsection{Shapes}

We define shapes by the following inductive rules:

1) $I$ is a shape

2) 1 is a shape 
3) if $S$ and $T$ are shapes then so is $S \otimes T$

4) if $S$ and $T$ are shapes then so is $[S, T]$

Thus shapes are formal objects built from $1, I, \otimes$ and $[$,$] .$

We assign to each shape $T$ a variable set $v(T)$ which may be considered as a list of +'s and -'s, defined inductively as follows:

1) $v(I)=\emptyset$

2) $v(1)=\{+\}$

3) $v(T \otimes S)=v(T) \amalg v(S)$

4) $v([T, S])=v(T)^{\mathrm{op}} \amalg v(S)$

Here $\amalg$ is the concatenation of lists and $v(T)^{\text {op }}$ is $v(T)$ with all signs reversed. Kelly and Mac Lane write

$$
\begin{gathered}
v(T) \coprod v(S)=v(T) \hat{+} v(S) \\
v(T)^{\mathrm{op}} \coprod v(S)=v(T) \tilde{+} v(S)
\end{gathered}
$$

and call these the ordered sum and twisted sum respectively. The sign of each variable is called its variance.

In fact we only need the strict monoidal version of this theory. That is, we put

$$
(T \otimes S) \otimes R=T \otimes(S \otimes R)
$$

and

$$
T \otimes I=T
$$

For example,

$$
[[1,1] \otimes 1 \otimes 1, I] \otimes 1
$$

is a shape with

$$
v(T)=\{+,-,-,-,+\}
$$

\subsection{Graphs}

A graph $T \longrightarrow S$ is defined to be a fixed point free pairing of the variables in $T$ and $S$ such that paired elements have opposite variances in $v(T)^{\text {op }} \amalg v(S)$. (Kelly and Mac Lane refer to such paired elements as "mates".) Equivalently, this is a bijection between the +'s and the -'s in $v(T)^{\text {op }} \amalg v(S)$.

For example, the following is a graph

$$
[[1,1] \otimes 1 \otimes 1, I] \otimes 1 \longrightarrow[1 \otimes 1,1 \otimes[1,1]]
$$

showing variances: 


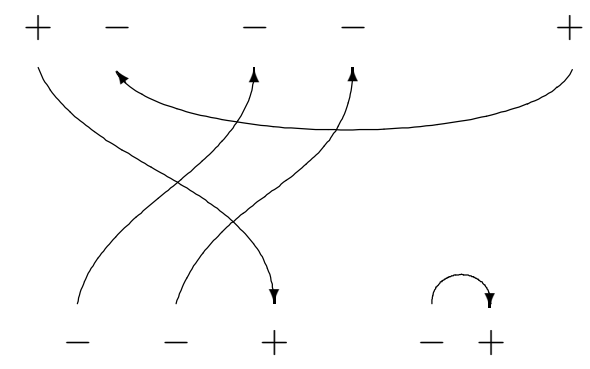

Graphs are composed in the obvious way, so that shapes and graphs form a category $G$. Moreover, $G$ has the structure of a closed category as follows. $\otimes$ and $[$,$] are defined on graphs in the obvious way, and the constraints$ are given by the following graphs:

$a$
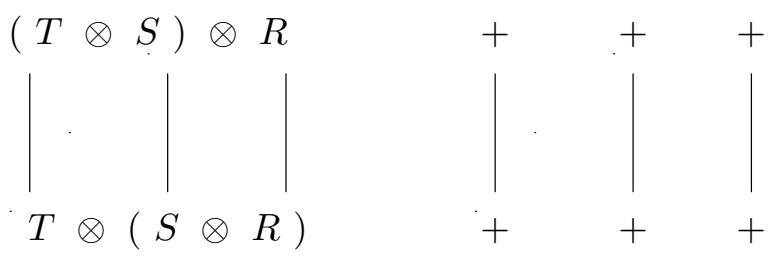

$b$
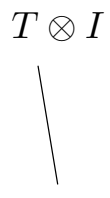

$T$

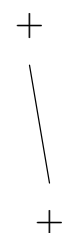

$c$

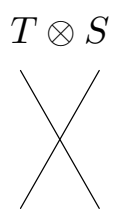

$S \otimes T$

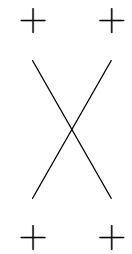

$d$
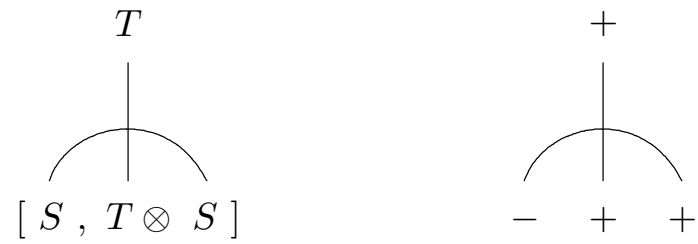

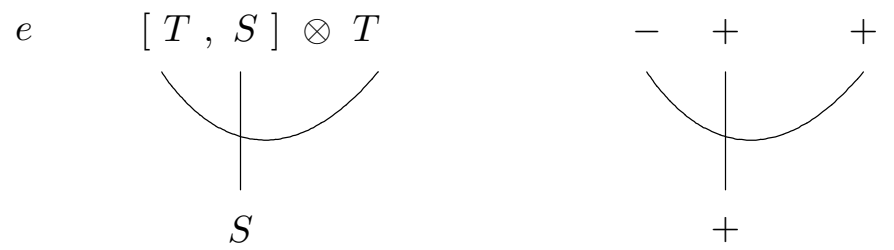

The diagrams on the right give variances, showing that these are indeed graphs; note that in the twisted sum the variances of the domain are reversed. For the strict monoidal version we have $a=1$ and $b=1$.

Observe that we realise Kelly-Mac Lane graphs as pictorial graphs by joining paired objects up with an edge. In the diagrams above, the objects are in fact shapes, so the drawn edges in fact represent multiple edges as necessary.

In fact there is a notion of graphs labelled in a category $\mathbb{C}$ (see [3]); these are the morphisms of a category which we will call $K \mathbb{C}$. Then the graphs above may be considered as graphs labelled in the category $\mathbf{1}$. So for consistency we write $G=K \mathbf{1}$.

\subsection{Allowable morphisms}

The allowable morphisms are then defined to be the smallest class of morphisms of $K \mathbf{1}$ satisfying the following conditions:

1) For any $T, S, R$ each of the following morphisms is in the class:

$$
\begin{aligned}
1 & : T \longrightarrow T \\
a & :(T \otimes S) \otimes R \longrightarrow T \otimes(S \otimes R) \\
a^{-1} & : T \otimes(S \otimes R) \longrightarrow(T \otimes S) \otimes R \\
b & : T \otimes I \longrightarrow T \\
b^{-1} & : T \longrightarrow T \otimes I \\
c & : T \otimes S \longrightarrow S \otimes T .
\end{aligned}
$$

2) For any $T, S$ each of the following morphisms is in the class:

$$
\begin{array}{ll}
d & : \quad T \longrightarrow[S, T \otimes S] \\
e & : \quad[T, S] \otimes T \longrightarrow S .
\end{array}
$$

3) If $f: T \longrightarrow T^{\prime}$ and $g: S \longrightarrow S^{\prime}$ are in the class so is

$$
f \otimes g: T \otimes S \longrightarrow T^{\prime} \otimes S^{\prime} .
$$

4) If $f: T \longrightarrow T^{\prime}$ and $g: S \longrightarrow S^{\prime}$ are in the class then so is

$$
[f, g]:\left[T^{\prime}, S\right] \longrightarrow\left[T, S^{\prime}\right] .
$$


5) If $f: T \longrightarrow S$ and $g: S \longrightarrow R$ are in the class then so is $g f: T \longrightarrow R$.

We write $A \mathbf{1}$ for the category of shapes and allowable morphisms.

The main theorem of $[8$ that we use is as follows:

Theorem 1.1 If $f: T \longrightarrow S$ and $g: S \longrightarrow R \in G$ are allowable then they are compatible, that is, composing them gives no closed loops.

For the proof (an induction over structure), see 8 .

\subsection{Duality}

Since $K 1$ is closed, given any graph

$$
\xi: S \otimes T \longrightarrow U \in K \mathbf{1}
$$

there is a unique dual

$$
\bar{\xi}: S \longrightarrow[T, U]
$$

so in particular, given a graph

$$
\alpha: S \longrightarrow T
$$

there is a unique dual

$$
\bar{\alpha}: I \longrightarrow[S, T]
$$

We will eventually be concerned with graphs of the form

$$
\alpha: A_{1} \otimes \cdots \otimes A_{k} \longrightarrow B
$$

it is sometimes convenient or indeed necessary to use the dual

$$
\bar{\alpha}: I \longrightarrow\left[A_{1} \otimes \cdots \otimes A_{k}, B\right]
$$

and we may refer to either of these graphs as $\alpha$ when the exact form is not relevant.

\section{Informal description of trees}

We consider unlablled, 'combed' trees, with ordered nodes. For example the following is a tree: 


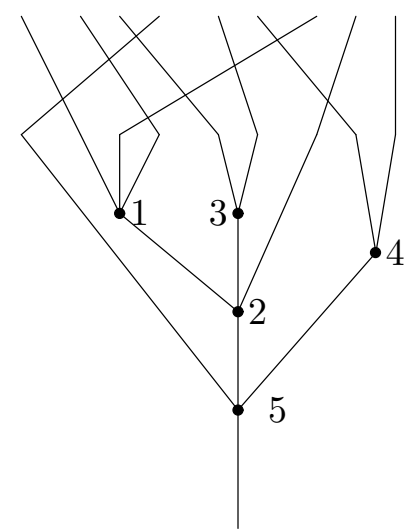

Explicitly, a tree $T=(T, \rho, \tau)$ consists of

i) A planar tree $T$

ii) A permutation $\rho \in \mathbf{S}_{l}$ where $l=$ number of leaves of $T$

iii) A bijection $\tau:\{$ nodes of $T\} \longrightarrow\{1,2, \ldots, k\}$ where $k=$ number of nodes of $T$; equivalently an ordering on the nodes of $T$.

Note that there is a 'null tree' with no nodes

\section{Formal description of trees}

In this section we give a formal description of the above trees, characterising them as connected graphs with no closed loops (in the conventional sense of 'graph'). This enables us, in Section 4 to express a tree as a morphism in $K 1$; it also enables us, in Section 7 to show that all allowable graphs of the correct shape arise in this way.

We consider a tree with $k$ nodes $N_{1}, \ldots, N_{k}$ where $N_{i}$ has $m_{i}$ inputs and one output. Let $N$ be a node with $\left(\sum_{i} m_{i}\right)-k+1$ inputs; $N$ will be used to represent the leaves and root of the tree.

Then a tree is given by a bijection

$\coprod_{i}\left\{\right.$ inputs of $\left.N_{i}\right\} \coprod\{$ output of $N\} \longrightarrow \coprod_{i}$ \{output of $\left.N_{i}\right\} \coprod$ inputs of $\left.N\right\}$ 
since each input of a node is either connected to a unique output of another node, or it is a leaf, that is, input of $N$. Similarly each output of a node is either attached to an input of another node, or it is the root, that is, output of $N$.

We express this formally as follows.

Lemma 3.1 Let $T$ be a tree with nodes $N_{1}, \ldots, N_{k}$, where $N_{i}$ has inputs $\left\{x_{i 1}, \ldots, x_{i m_{i}}\right\}$ and output $x_{i}$. Let $N$ be a node with inputs $\left\{z_{1}, \ldots, z_{l}\right\}$ and output $z$, with

$$
l=\left(\sum_{i=1}^{k} m_{i}\right)-k+1 .
$$

Then $T$ is given by a bijection

$$
\alpha: \coprod_{i}\left\{x_{i 1}, \ldots, x_{i m_{i}}\right\} \coprod\{z\} \longrightarrow \coprod_{i}\left\{x_{i}\right\} \coprod\left\{z_{1}, \ldots, z_{l}\right\} .
$$

Proof. We construct the bijection $\alpha$.

Consider $x_{i j}$ on the left hand side. This is the $j$ th input of $N_{i}$, which is either

i) joined to the output of a unique $N_{r}$, in which case $\alpha\left(x_{i j}\right)=x_{r}$, or

ii) the $p$ th leaf of the tree, in which case $\alpha\left(x_{i j}\right)=z_{p}$.

Finally, $z$ is the root of the tree, so must be the output of a unique $N_{r}$, so $\alpha(z)=x_{r}$.

For the inverse, consider $x_{r}$ on the right hand side. This is the output of the $r$ th node, so is either

i) joined to the $j$ th input of a unique $N_{i}$, in which case $\alpha^{-1}\left(x_{r}\right)=\alpha\left(x_{i j}\right)$, or

ii) is the root of the tree, in which case $\alpha^{-1}\left(x_{r}\right)=z$.

Each $z_{r}$ is a leaf of the tree, so must be the $j$ th input of a unique $N_{i}$, so $\alpha^{-1}\left(z_{r}\right)=x_{i j}$.

$\alpha^{-1}$ thus defined is inverse to $\alpha$, so $\alpha$ is a bijection.

Note that if $k=0$ we have the null tree with no nodes; then $l=1$ and $N$ has one input $z_{1}$. Then the bijection $\alpha$ is given by $\alpha(z)=z_{1}$.

For example, consider 

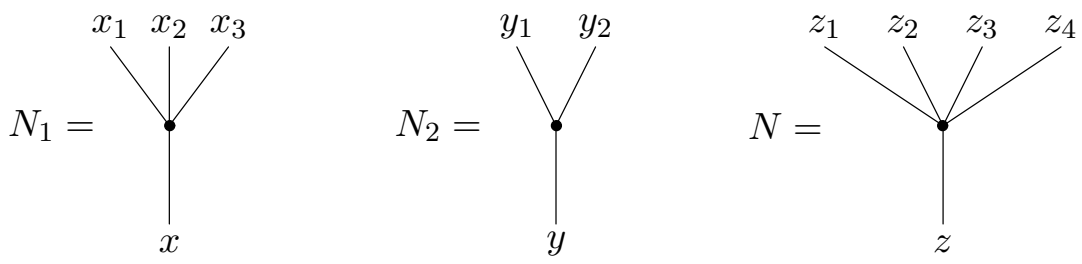

Then a tree

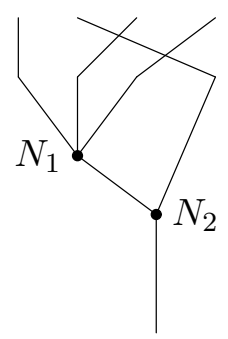

is given by the following bijection:

$$
\begin{aligned}
\left\{x_{1}, x_{2}, x_{3}, y_{1}, y_{2}, z\right\} & \longrightarrow\left\{x, y, z_{1}, z_{2}, z_{3}, z_{4}\right\} \\
x_{1} & \longmapsto z_{1} \\
x_{2} & \longmapsto z_{3} \\
x_{3} & \longmapsto z_{4} \\
y_{1} & \longmapsto x \\
y_{2} & \longmapsto z_{2} \\
z & \longmapsto y .
\end{aligned}
$$

For the converse, every such bijection gives a graph, but it is not necessarily a tree. For example

$$
\begin{aligned}
x_{1} & \longmapsto y \\
x_{2} & \longmapsto z_{3} \\
x_{3} & \longmapsto z_{4} \\
y_{1} & \longmapsto z_{2} \\
y_{2} & \longmapsto z_{2} \\
z & \longmapsto z_{1}
\end{aligned}
$$

gives the following graph: 


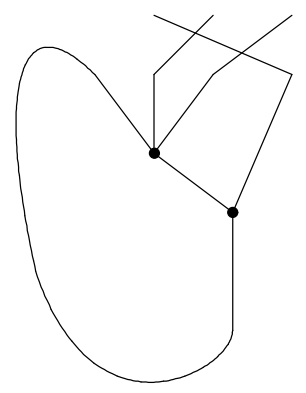

So we need to ensure that the resulting graph has no closed loops; the use of the 'formal' node $N$ then ensures connectedness. We express this formally as follows.

Lemma 3.2 Let $N_{1}, \ldots, N_{k}, N$ be nodes where $N_{i}$ has inputs $\left\{x_{i 1}, \ldots, x_{i m_{i}}\right\}$ and output $x_{i}$, and $N$ has inputs $\left\{z_{1}, \ldots, z_{l}\right\}$ and output $z$, with $l=\left(\sum_{i=1}^{k} m_{i}\right)-$ $k+1$. Let $\alpha$ be a bijection

$$
\coprod_{i}\left\{x_{i 1}, \ldots, x_{i m_{i}}\right\} \coprod\{z\} \longrightarrow \coprod_{i}\left\{x_{i}\right\} \coprod\left\{z_{1}, \ldots, z_{l}\right\} .
$$

Then $\alpha$ defines a graph with nodes $N_{1}, \ldots, N_{k}$.

Lemma 3.3 Let $\alpha$ be a graph as above. Then $\alpha$ has a closed loop if and only if there is a non-empty sequence of indices

$$
\left\{t_{1}, \ldots, t_{n}\right\} \subseteq\{1, \ldots, k\}
$$

such that for each $2 \leq j \leq n$

$$
\alpha\left(x_{t_{j} b_{j}}\right)=x_{t_{j-1}}
$$

for some $1 \leq b_{j} \leq m_{j}$, and

$$
\alpha\left(x_{t_{1} b_{1}}\right)=x_{t_{n}}
$$

for some $1 \leq b_{1} \leq m_{1}$.

Proof. A closed loop in $\alpha$ is a sequence of nodes

$$
\left\{N_{t_{1}}, \ldots, N_{t_{n}}\right\}
$$

such that for each $2 \leq j \leq n, N_{t_{j}}$ is joined to $N_{t_{j-1}}$, and also $N_{t_{1}}$ is joined to $N_{t_{n}}$. 
That is, for each $2 \leq j \leq n$, some leaf of $N_{t_{j}}$ is joined to the root of $N_{t_{j-1}}$, and also some leaf of $N_{t_{1}}$ is joined to $N_{t_{n}}$. This is precisely the case described formally in the Lemma, with the $b_{j}$ giving the leaves in question.

For example in the above case we have

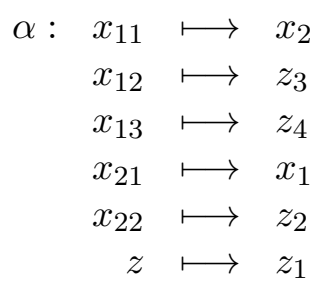

which has a loop given by indices $\{1,2\}$, since

$$
\alpha\left(x_{21}\right)=x_{1} \text { and } \alpha\left(x_{11}\right)=x_{2} .
$$

Note that a graph with no nodes cannot satisfy the above condition since the sequence $\left\{N_{t_{1}}, \ldots, N_{t_{n}}\right\}$ is required to be non-empty.

Corollary 3.4 A tree with nodes $N_{1}, \ldots, N_{k}$ is precisely a bijection $\alpha$ as in Lemma 3.2, such that there is no sequence of indices as in Lemma 3.3.

Proof. $\quad \alpha$ defines a graph; this is a tree if and only if there is no closed loop. Note that if $k=0$ we have a bijection

$$
\alpha:\{z\} \longrightarrow\left\{z_{1}\right\}
$$

that is, the null tree.

\section{Trees as morphisms in $K 1$}

We now show how trees may be expressed as graphs. Here we consider unlabelled trees; the labelled version follows easily.

Let $\mathbf{1}$ be the category with just one object and one (identity) morphism. We write the single object of $\mathbf{1}$ as 1 . Then we express a node of a tree as the following object in $K \mathbf{1}$

$$
X_{m}=[1 \otimes \ldots \otimes 1,1]=\left[1^{\otimes m}, 1\right]
$$

where $m$ is the number of inputs of the node.

Now consider a tree $T$ with (ordered) nodes $N_{1}, \ldots N_{k}$ where $N_{i}$ has $m_{i}$ inputs. We show that this tree may be represented as a morphism

$$
X_{m_{1}} \otimes \ldots \otimes X_{m_{k}} \stackrel{\xi_{T}}{\longrightarrow} X_{l} \in K \mathbf{1}
$$

using the formal description of trees as in Section 3 . 
Lemma 4.1 Let $T$ be a tree with $N_{1}, \ldots, N_{k}$ be nodes where $N_{i}$ has inputs $\left\{x_{i 1}, \ldots, x_{i m_{i}}\right\}$ and output $x_{i}$. Then $T$ is given by a morphism

$$
\xi_{T}: X_{m_{1}} \otimes \ldots \otimes X_{m_{k}} \longrightarrow X_{l} \in K \mathbf{1}
$$

where $l=\left(\sum_{i=1}^{k} m_{i}\right)-k+1$. Note that if $k=0$ then the left hand side of the above expression becomes $I$.

Proof. Recall that a graph $\xi_{T}$ as above is precisely a bijection from the -'s to the +'s in the twisted sum

$$
v\left(X_{m_{1}} \otimes \ldots \otimes X_{m_{k}}\right) \tilde{+} v\left(X_{l}\right) .
$$

By Lemma 3.1, $T$ is given by a bijection

$$
\coprod_{i}\left\{x_{i 1}, \ldots, x_{i m_{i}}\right\} \coprod\{z\} \longrightarrow \coprod_{i}\left\{x_{i}\right\} \coprod\left\{z_{1}, \ldots, z_{l}\right\} .
$$

Observe that the elements of the left hand side of this expression are precisely the -'s in the twisted sum above, and those of the right hand side are precisely the +'s.

As in Section 3 the idea is that a tree is constructed by identifying each node output with the node input to which it is joined, unless it is the root; similarly each input is identified with a node output unless it is a leaf. This identification gives the mates in the graph $\xi_{T}$, where the codomain $X_{l}$ is representing the leaves and the root of the tree $T$.

For example the following tree as described in Section [3]

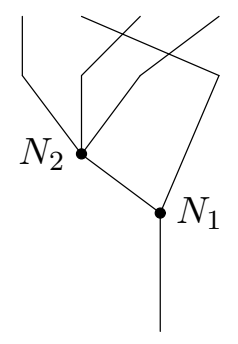

is be expressed as the following morphism in $K \mathbf{1}$ 


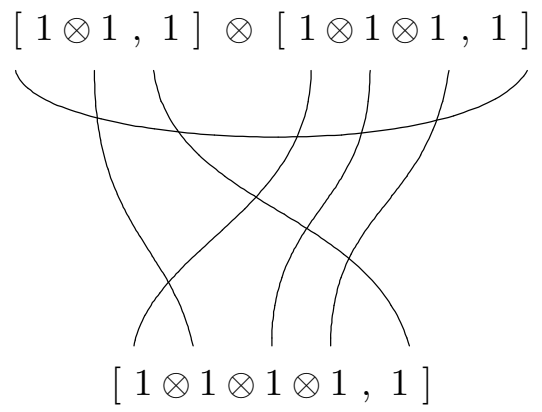

and the following representation giving variances shows that this is indeed a graph:

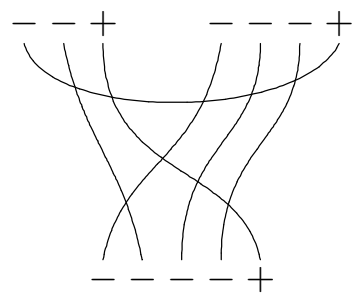

Formally, the graph for a tree $T$ as above is given as follows. We write

$$
\begin{aligned}
X_{m_{i}} & =\left[A_{i 1} \otimes \ldots \otimes A_{i m_{i}}, A_{i}\right] \\
X_{l} & =\left[B_{1}, \otimes \ldots \otimes B_{l},, B\right]
\end{aligned}
$$

where each $A_{i j}, A_{i}, B_{i}, B=1$ and in the twisted sum we have variances

$$
\begin{array}{cc}
v\left(A_{i j}\right)=+, & v\left(A_{i}\right)=- \\
v\left(B_{p}\right)=-, & v(B)=+.
\end{array}
$$

Then the graph $\xi_{T}$ is given as follows.

- considering node inputs

For each $i, j$, either

i) the $j$ th input of $N_{i}$ is joined to the output of $N_{r}$, say, in which case $A_{i j}$ is the mate of $A_{r}$, or

ii) the $j$ th input of $N_{i}$ is the $p$ th leaf of the tree $T$, in which case $A_{i j}$ is the mate of $B_{p}$ in $\xi_{T}$.

- considering node outputs 
For each $r$, either

i) the output of $N_{r}$ is the root of the tree, in which case $B_{r}$ is the mate of $B$, or

ii) the output of $N_{r}$ is joined to the $j$ th input of $N_{i}$, say, in which case $A_{r}$ is the mate of $A_{i j}$.

Note that the null tree

$$
\mid
$$

is a graph $I \stackrel{\xi}{\longrightarrow} X_{1}$ as follows:

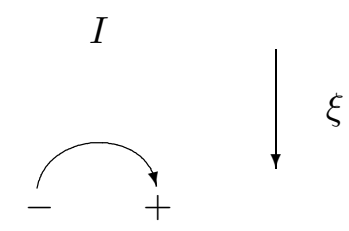

So we have shown that every tree is given by a graph in $K \mathbf{1}$; in Section 6 we show that any such graph is allowable. The proof is by induction, and the following section enables us to makes the induction step.

\section{Composition of trees}

We now discuss two ways of composing trees:

1) leaf-root composition in which a leaf of one tree is attached to the root of another, for example

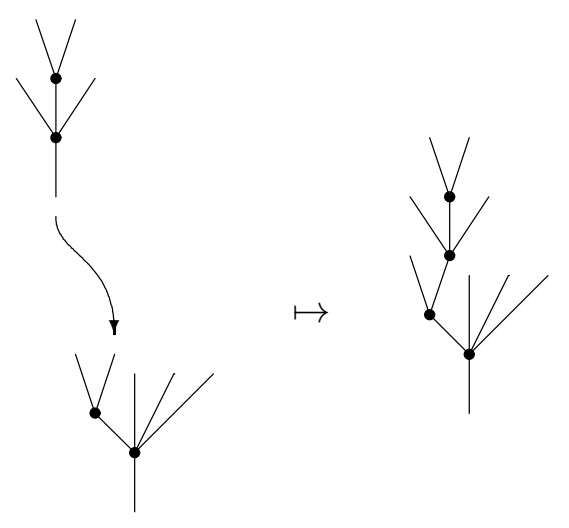


2) node-replacement composition in which a node of one tree is replaced by another tree, for example

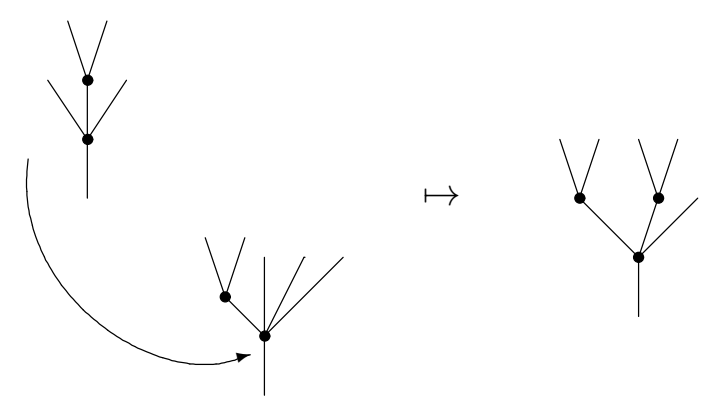

In the first case the inputs of the tree are considered to be the leaves, and the output the root; note that an issue of node-ordering arises, so that this 'composition' is not associative. However, it facilitates the induction argument in Section [6, which is why we discuss it here.

In the second case the inputs are the nodes, and the output a node with one input edge for each leaf of the tree. This form of composition is used in later work ([3]) when we describe the construction of opetopes in the framework of Kelly-Mac Lane graphs.

We show how each of these forms of composition arises for trees represented as graphs as in Section 4

Recall that a tree is expressed as a morphism

$$
X_{m_{1}} \otimes \cdots \otimes X_{m_{k}} \longrightarrow X_{l} \in K \mathbf{1}
$$

Now in general, given any morphisms in $K \mathbf{1}$

$$
\begin{aligned}
& B_{1} \otimes \cdots \otimes B_{n} \stackrel{f}{\longrightarrow} A_{p} \\
& A_{1} \otimes \cdots \otimes A_{m} \stackrel{g}{\longrightarrow} A
\end{aligned}
$$

for some $1 \leq p \leq m$, we may form the composite

$$
f \circ(1 \otimes \cdots \otimes 1 \otimes g \otimes 1 \otimes \cdots \otimes 1)
$$

which we write as

$$
g \circ_{p} f: A_{1} \otimes \cdots \otimes A_{j-1} \otimes B_{1} \otimes \cdots \otimes B_{n} \otimes A_{p+1} \otimes \cdots \otimes A_{m} \longrightarrow A .
$$

Note that if $p$ is evident from the context we simply write $g \circ f$.

This composition gives node-replacement composition of trees. Consider trees $S, T$ with graphs

$$
\xi_{S}: X_{s_{1}} \otimes \cdots \otimes X_{s_{n}} \longrightarrow X_{l}
$$




$$
\xi_{T}: X_{t_{1}} \otimes \cdots \otimes X_{t_{m}} \longrightarrow X_{k}
$$

Then $\mathrm{S}$ may be composed at the $p$ th node of $T$ if the number of leaves of $S$ equals the number of inputs of the $p$ th node, that is, if $X_{l}=X_{t_{p}}$. Then the graph for the composite tree is given by

$$
\xi_{S} \circ_{p} \xi_{T} .
$$

For example as above, suppose we have $p=2$ and

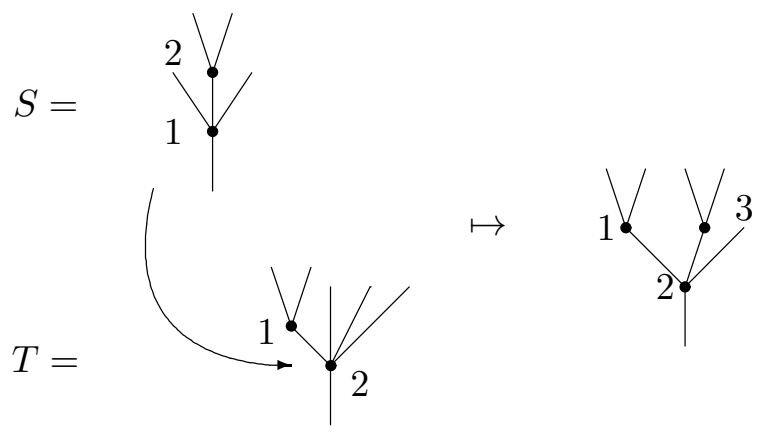

then we express this with graphs as follows
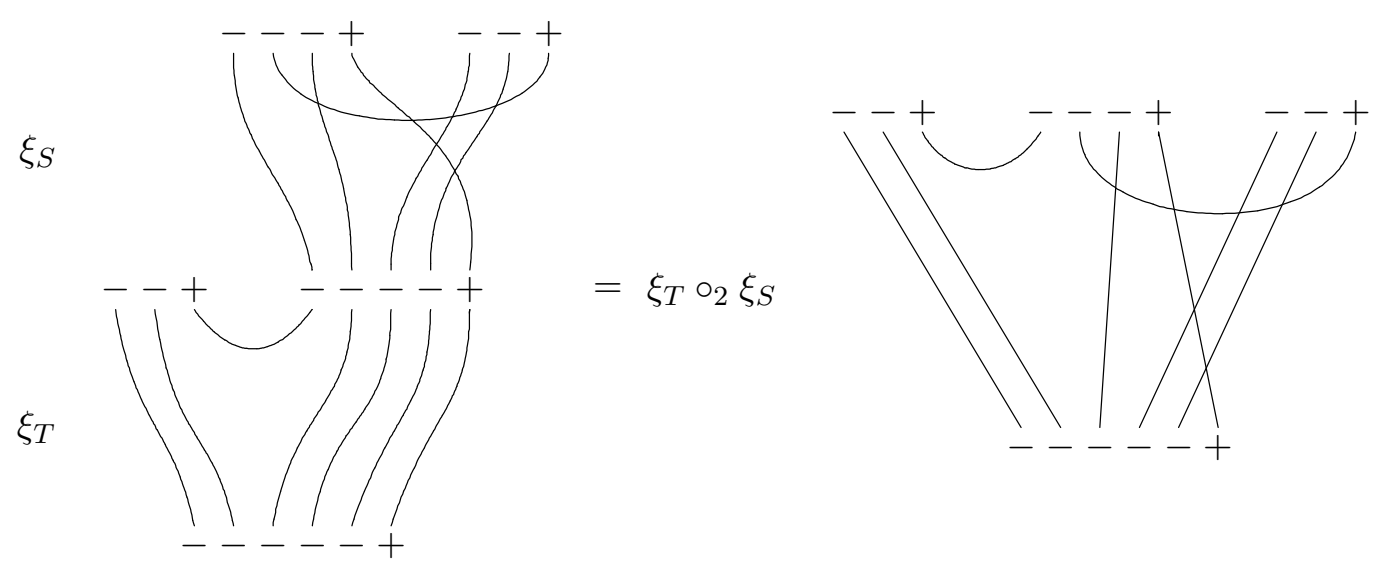

In fact, considering the dual forms $\bar{\xi}_{S}$ and $\bar{\xi}_{T}$, we see that this composite may also be expressed by means of a 'composition graph' $\xi$ as follows. We have

$$
\begin{gathered}
\bar{\xi}_{S}: I \longrightarrow\left[X_{s_{1}} \otimes \cdots \otimes X_{s_{n}}, X_{l}\right] \\
\bar{\xi}_{T}: I \longrightarrow\left[X_{t_{1}} \otimes \cdots \otimes X_{t_{m}}, X_{k}\right]
\end{gathered}
$$


Then $\xi$ is a graph

$$
\begin{gathered}
{\left[X_{t_{1}} \otimes \cdots \otimes X_{t_{m}}, X_{k}\right] \otimes\left[X_{s_{1}} \otimes \cdots \otimes X_{s_{n}}, X_{l}\right]} \\
\downarrow \\
\downarrow \\
{\left[X_{t_{1}} \otimes \cdots \otimes X_{t_{p-1}} \otimes X_{s_{1}} \otimes \cdots \otimes X_{s_{n}} \otimes X_{t_{p+1}} \otimes \cdots \otimes X_{t_{m}}, X_{k}\right]}
\end{gathered}
$$

where $X_{l}$ is joined to $X_{t_{p}}$ in the domain, and for all other $j, X_{j}$ in the domain is joined to $X_{j}$ in the codomain.

We now consider leaf-root composition. Consider trees $S, T$ as above. We seek to attach the root of $S$ to the $q$ th leaf of $T$, and we adopt the convention that the nodes of $S$ are then listed before those of $T$ in the final tree.

This is achieved in $K \mathbf{1}$ by placing the graphs $\xi_{S}$ and $\xi_{T}$ side by side, that is, taking their tensor product, and composing the result with a 'composition graph' that joins up the correct leaf and root as required. We write

$$
\begin{aligned}
X_{l} & =\left[A_{1} \otimes \cdots \otimes A_{l}, A\right] \\
X_{k} & =\left[B_{1} \otimes \cdots \otimes B_{k}, B\right] \\
X_{l+k-1} & =\left[C_{1} \otimes \cdots \otimes C_{l+k-1}, C\right]
\end{aligned}
$$

and the 'composition graph' as

$$
\xi: X_{l} \otimes X_{k} \longrightarrow X_{l+k-1} .
$$

The idea is that the leaves of $S$ are inserted into the list of leaves of $T$ at the $q$ th place to give

$$
\left[B_{1} \otimes \cdots \otimes B_{q-1} \otimes A_{1} \otimes \cdots \otimes A_{l} \otimes B_{q+1} \otimes \cdots \otimes B_{k}, B\right]
$$

so the composition graph $\xi$ is given as follows:

i) the mate of $A$ is $B_{q}$

ii) the mate of $B$ is $C$

iii) for $1 \leq i \leq l$ the mate of $A_{i}$ is $C_{q+i-1}$

iv) for $1 \leq i \leq q-1$ the mate of $B_{i}$ is $C_{i}$

v) for $q+1 \leq i \leq k$ the mate of $B_{i}$ is $C_{l+i-1}$.

For example, suppose we have $q=2$ with 


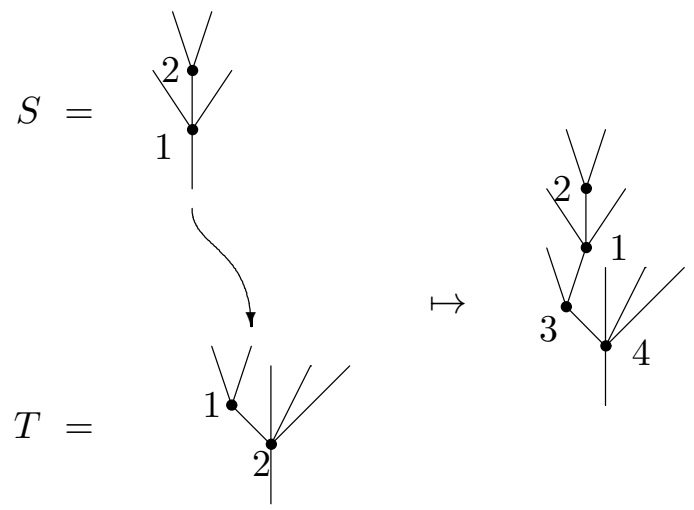

then this is represented by the following graph in $K \mathbf{1}$ :
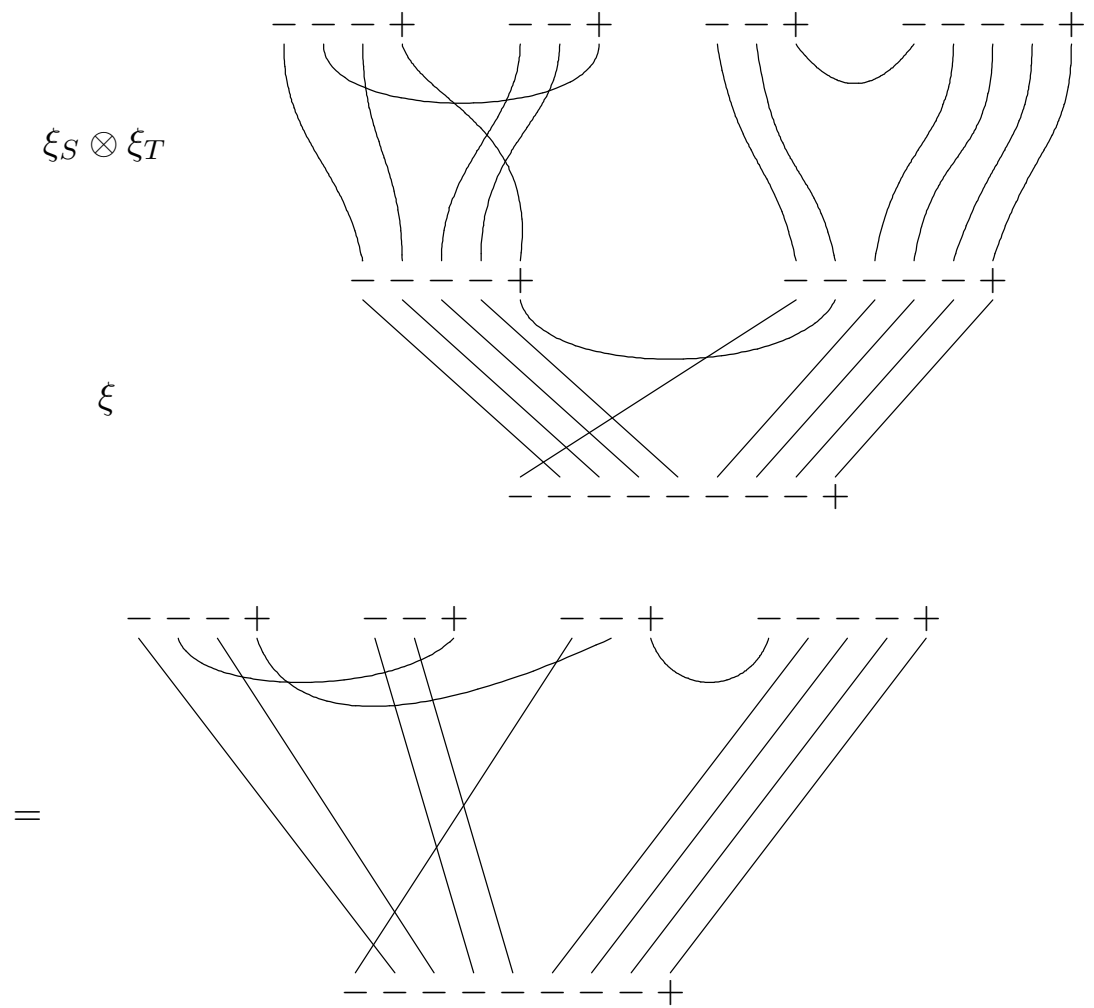

Note that we could adopt a different convention for ordering the nodes of the composite tree, using $\xi_{T} \otimes \xi_{S}$. Of course, neither convention yields an associative composition, but since we are not at this time trying to form a category (or multicategory) of such trees, we do not pursue this matter here. 


\section{The graph of a tree is allowable}

We have shown how any tree is represented by a graph. We now show that any such graph is allowable.

Proposition 6.1 Given a tree $T$ as above, the graph $\xi_{T}$ is allowable.

Proof. By induction on the height of trees. Here the height of a tree is the maximum number of nodes on any path from a leaf to the root. A tree of height 0 is the null tree

$$
\mid
$$

represented by the graph

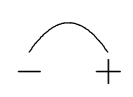

which is the morphism

$$
I \stackrel{d_{I 1}}{\longrightarrow}[1, I \otimes 1]=[1,1]
$$

which is allowable.

A tree of height 1 is just a node

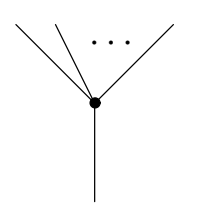

which is represented by an identity graph

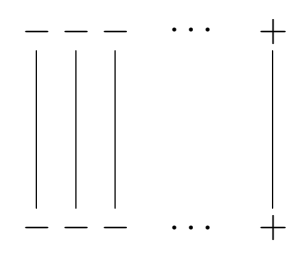


which is allowable.

A tree of height $h \geq 1$ may be considered as a composite

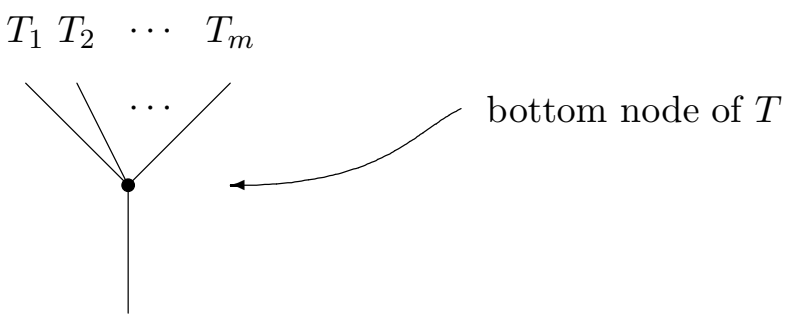

where the $T_{i}$ are subtrees of $T$; by construction they have height $\leq h$. So by induction each of these is represented by an allowable graph.

It is therefore sufficient to show that leaf-root composition of allowable graphs gives an allowable graph. Note that leaf-root composition as defined in Section 5 will not necessarily give the correct node ordering on the final tree; however, this can be achieved by composing with symmetries as necessary. This will not affect the allowability of the graph since symmetries are allowable graphs, and composites of allowable graphs are allowable.

Furthermore, since tensors and composites of allowable graphs are allowable, it is sufficient to show that all 'composition graphs' $\xi$ as defined in Section [5] are allowable.

Since any permutation may be written as a composite of transpositions, and is therefore allowable, we may assume without loss of generality that $q=1$ in the composition. So it is sufficient to show that any graph $\xi$ of the following form is allowable. Writing

$$
\begin{aligned}
X_{m_{1}} & =\left[A_{1} \otimes \cdots \otimes A_{m_{1}}, A\right] \\
X_{m_{2}} & =\left[B_{1} \otimes \cdots \otimes B_{m_{2}}, B\right] \\
X_{m_{1}+m_{2}-1} & =\left[C_{1} \otimes \cdots \otimes C_{m_{1}+m_{2}-1}, C\right]
\end{aligned}
$$

then

$$
\xi: X_{m_{1}} \otimes X_{m_{2}} \longrightarrow X_{m_{1}+m_{2}-1}
$$

is given as follows.

i) the mate of $A$ is $B_{1}$

ii) the mate of $B$ is $C$

iii) for all $1 \leq i \leq m_{1}$ the mate of $A_{i}$ is $C_{i}$

iv) for $2 \leq i \leq m_{2}$ the mate of $B_{i}$ is $C_{m_{1}+i-1}$. 
So $\xi$ has the form

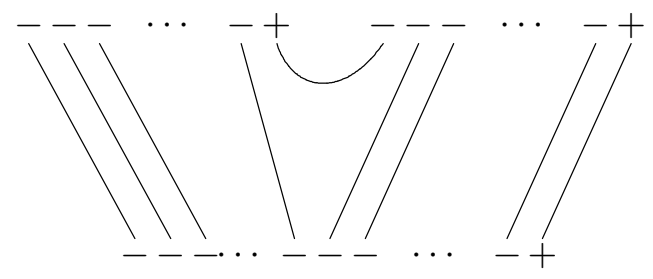

Writing

$$
\begin{aligned}
& A_{1} \otimes \cdots \otimes A_{m_{1}}=\bar{A} \\
& B_{2} \otimes \cdots \otimes B_{m_{2}}=\bar{B}
\end{aligned}
$$

we may abbreviate this as

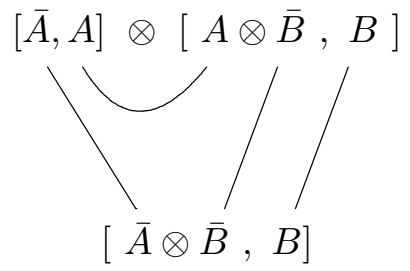

which may be written as the following composite of allowable graphs:

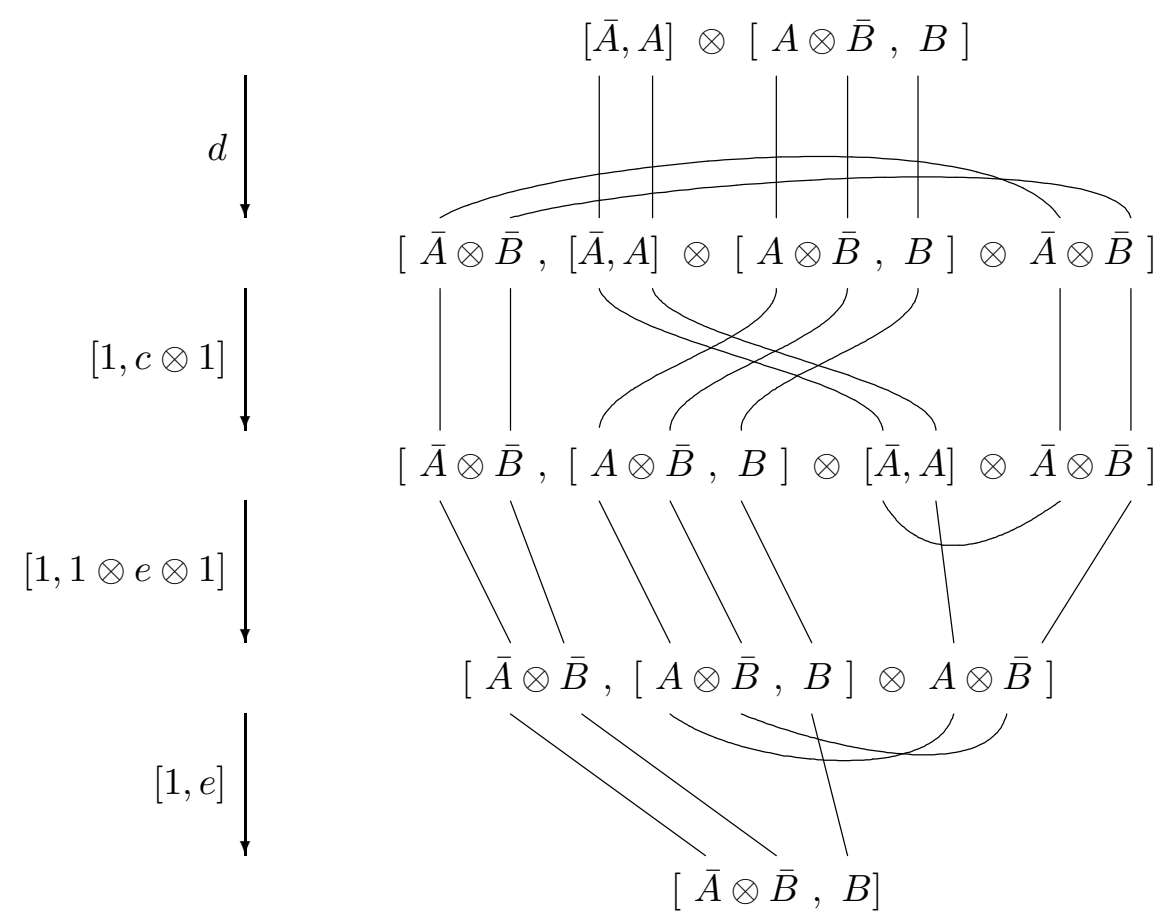




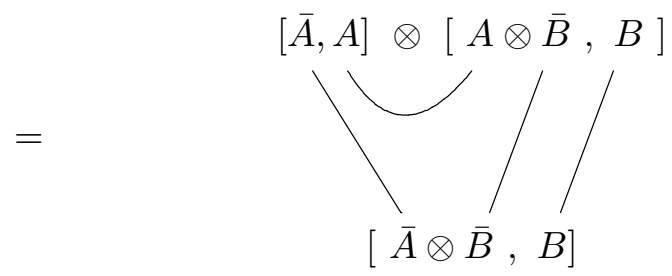

so $\xi$ is allowable as required.

\section{Every allowable graph is a tree}

We have seen that every tree is represented by a unique graph, and that this graph is allowable. In this section we prove the converse, that every allowable graph of the correct shape represents a unique tree.

We now use the characterisation of trees as in Section 3 . As in that section, for the converse we see that every morphism

$$
X_{m_{1}} \otimes \cdots \otimes X_{m_{k}} \longrightarrow X_{l} \in K \mathbf{1}
$$

gives a graph but that it is not necessarily a tree; we need to ensure that the resulting graph has no closed loops. We copy Lemmas 3.2 and 3.3 "translating" them into the language of closed categories. Note that the word 'graph' is used in the ordinary sense; for clarity we refer to KellyMac Lane graphs as 'morphisms in $K \mathbf{1}$ '.

Lemma 7.1 Let $N_{1}, \ldots, N_{k}$ be nodes where $N_{i}$ has inputs

$$
\left\{A_{i 1}, \ldots, A_{i m_{i}}\right\}
$$

and output $x_{i}$. Let $\xi$ be a morphism

$$
\xi: X_{m_{1}} \otimes \ldots \otimes X_{m_{k}} \longrightarrow X_{l} \in K \mathbf{1}
$$

where $l=\left(\sum_{i=1}^{k} m_{i}\right)-k+1$. Then $\xi$ defines a graph with nodes $N_{1}, \ldots, N_{k}$.

Lemma 7.2 Let $\xi$ be a graph as above. Then $\xi$ has a closed loop if and only if there is a non-empty set of indices

$$
\left\{t_{1}, \ldots, t_{n}\right\} \subseteq\{1, \ldots, k\}
$$

such that for each $2 \leq j \leq n$ the mate of $A_{t_{j-1}}$ under $\xi$ is $A_{t_{j} b_{j}}$ and the mate of $A_{t_{n}}$ is $A_{t_{1} b_{1}}$ for some $1 \leq b_{j} \leq m_{j}$. 
Proposition 7.3 If there is a set of indices $\left\{t_{1}, \ldots t_{n}\right\}$ as above then $\xi$ is not allowable.

Corollary 7.4 Let $\xi$ be a morphism as above. Then $\xi$ is a tree if and only if it is allowable.

To prove this we will use Theorem 1.1 (Theorem 2.2 of [8]) which states that if two composable morphisms are allowable then they are compatible, that is, composing them does not result in any closed loops. So to show that $\xi$ as above is not allowable, we aim to construct an allowable morphism $\eta$ such that $\eta$ and $\xi$ are not compatible. The following lemma provides us with such a morphism.

Lemma 7.5 Write $X_{k}=\left[A_{1} \otimes \cdots \otimes A_{k}, A\right]$ with $A_{i}, A=1$ and let $1 \leq p \leq k$.

Then there is an allowable morphism

$$
\theta_{p}:\left[A_{1} \otimes \cdots \otimes A_{p-1} \otimes A_{p+1} \otimes \cdots \otimes A_{k}, I\right] \longrightarrow X_{k}
$$

with graph

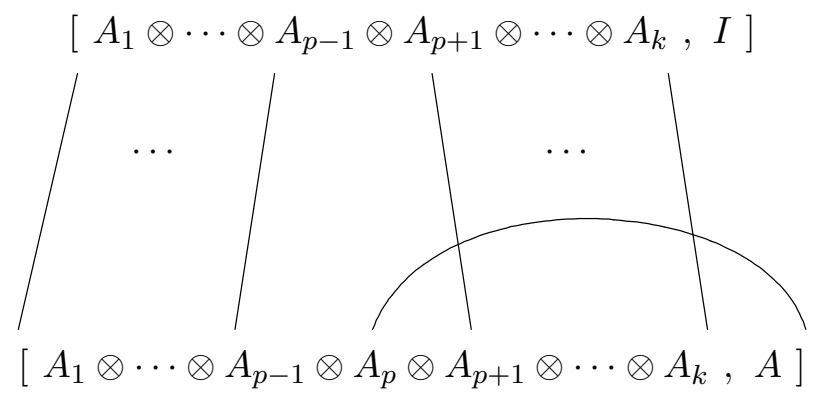

Proof. Write $Y=A_{1} \otimes \cdots \otimes A_{p-1} \otimes A_{p+1} \otimes \cdots \otimes A_{k}$. Since symmetries are allowable, it is sufficient to exhibit an allowable morphism

$$
[Y, I] \longrightarrow[Y \otimes 1,1]
$$

with underlying graph

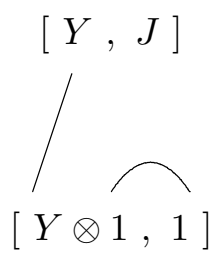


We have the following composite of allowable morphisms:

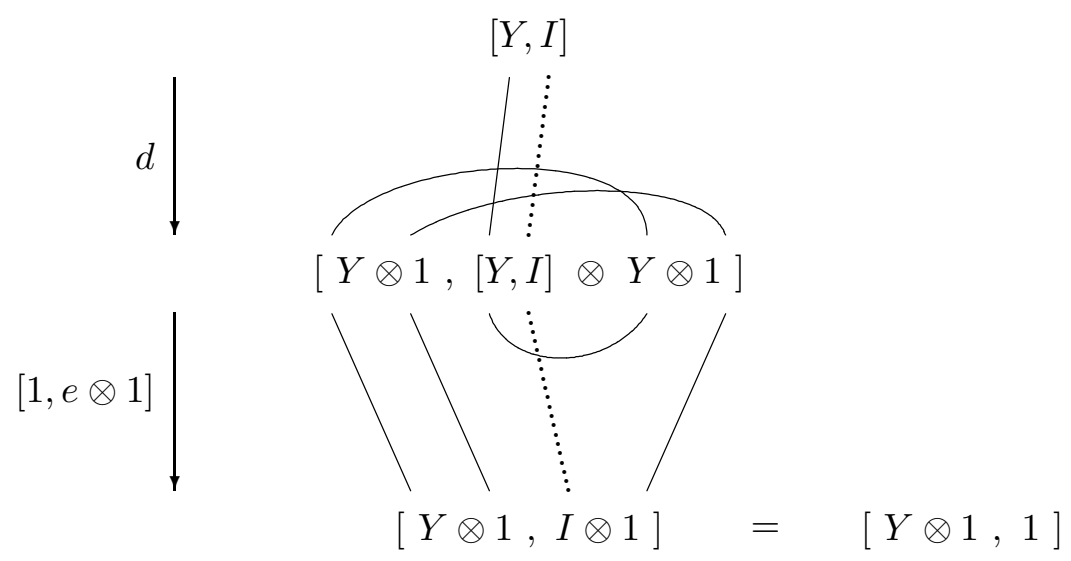

which has the underlying graph as required; since composites of allowable morphisms are allowable, the composite is allowable.

Proof of Proposition 7.3, To show that

$$
\xi: X_{m_{1}} \otimes \cdots \otimes X_{m_{k}} \longrightarrow X_{l}
$$

is not allowable we construct an allowable morphism

$$
\eta: T \longrightarrow X_{m_{1}} \otimes \cdots \otimes X_{m_{k}}
$$

such that $\eta$ and $\xi$ are not compatible, that is, composing them produces a closed loop.

We aim to construct $\eta$ in such a way that for each $1 \leq j \leq n$ the mate of $A_{t_{j}}$ is $A_{t_{j} b_{j}}$ so that in the composite graph we have the following closed loop:

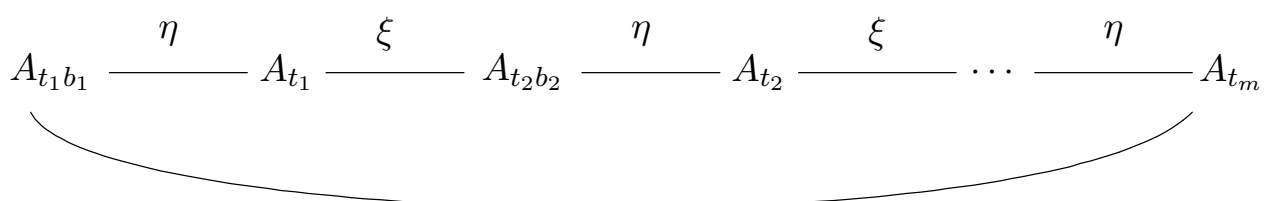

We use morphisms of the form $\theta_{p}$ as given in Lemma 7.5 .

Put $T=Y_{1} \otimes \cdots \otimes Y_{k}$ where

$$
Y_{i}=\left[A_{t_{j}}, \otimes \cdots \otimes A_{t_{j}\left(b_{j}-1\right)} \otimes A_{t_{j}\left(b_{j}+1\right)} \otimes \cdots \otimes A_{t_{j} m_{i}}, I\right]
$$


if $i=t_{j}$ for some $1 \leq j \leq n$, and

$$
Y_{i}=X_{m_{i}}
$$

We define $\eta$ as a tensor product

$$
f_{1} \otimes \cdots \otimes f_{k}: Y_{1} \otimes \cdots \otimes Y_{k} \longrightarrow X_{m_{1}} \otimes \cdots \otimes X_{m_{k}}
$$

where

$$
f_{i}= \begin{cases}\theta_{b_{j}} & \text { if } i=t_{j} \text { for some } 1 \leq j \leq n \\ 1 & \text { otherwise }\end{cases}
$$

By Lemma 7.5 each $f_{i}$ is allowable, so $\eta$ is allowable.

Since the mate of $A_{t_{j}}$ under $\theta_{b_{j}}$ is $A_{t_{j} b_{j}}$ we have a closed loop as above, so $\eta$ and $\xi$ are not compatible. Since $\eta$ is allowable, it follows from Theorem 1.1 that $\xi$ is not allowable.

Finally we sum up the results of this section in the following proposition.

Proposition 7.6 A tree is a unique morphism of the form

$$
X_{m_{1}} \otimes \cdots \otimes X_{m_{k}} \longrightarrow X_{l} \in K \mathbf{1}
$$

and this morphism is allowable. Conversely, any such allowable morphism represents a unique tree.

Corollary 7.7 A tree is a unique allowable morphism of the form

$$
I \longrightarrow\left[X_{m_{1}} \otimes \cdots \otimes X_{m_{1}}, X_{l}\right] \in K \mathbf{1}
$$

Conversely, any such allowable morphism represents a unique tree.

Proof. Follows from the closed structure of $K \mathbf{1}$.

In order to make Proposition [7.6 and Corollary 7.7 more precise, we seek an equivalence between a 'category of trees' and a 'category of allowable morphisms'. In fact, trees of this form arise naturally by considering configurations for composing arrows of a symmetric multicategory. That is, they arise from the 'slicing' process as defined in [1] and [4; the trees then appear as arrows of the multicategory $I^{2+}$, and so as objects of $I^{3+}$, forming a category $\mathbb{C}_{3}$.

So we may consider the slice construction using the representation in closed categories. In considering this for constructing trees, we in fact deal with all the machinery used in constructing $k$-opetopes for all $k \geq 0$, since these are formed by iterating the construction. This is the subject of []. 


\section{References}

[1] John Baez and James Dolan. Higher-dimensional algebra III: $n$ categories and the algebra of opetopes. Adv. Math., 135(2):145-206, 1998. Also available via http://math.ucr.edu/home/baez.

[2] R. Blute. Linear logic, coherence and dinaturality. Theoretical Computer Science, 115:3-41, 1993.

[3] Eugenia Cheng. The theory of opetopes via Kelly-Mac Lane graphs, October 2002. E-print math. CT/0304288.

[4] Eugenia Cheng. Weak $n$-categories: opetopic and multitopic foundations, October 2002. E-print math.CT/0304277.

[5] Jean-Yves Girard. Linear logic: its syntax and semantics. In Laurent Regnier, Jean-Yves Girard, Yves Lafont, editors, Advances in Linear Logic, volume 222 of $L M S$, pages 1-42. Cambridge University Press, 1995.

[6] André Joyal and Ross Street. Braided tensor categories. Advances in Mathematics, 102:20-78, 1983.

[7] G. Kelly and M. Laplaza. Coherence for compact closed categories. Journal of Pure and Applied Algebra, 19:193-213, 1980.

[8] G. M. Kelly and S. Mac Lane. Coherence in closed categories. Journal of Pure and Applied Algebra, 1(1):97-140, 1971.

[9] Yves Lafont. From proof-nets to interaction nets. In Laurent Regnier Jean-Yves Girard, Yves Lafont, editor, Advances in Linear Logic, volume 222 of $L M S$, pages 225-247. Cambridge University Press, 1995.

[10] Paul-André Melliès. On double categories and multiplicative linear logic, June 1999. to appear in Mathematical Structures in Computer Science.

[11] Mei Chee Shum. Tortile Tensor Categories. PhD thesis, Macquarie University, New South Wales, November 1989. 